\title{
Modeling of occurrence frequencies of ion conics as a function of altitude and conic angle
}

\author{
W. Miyake ${ }^{1}$, T. Mukai ${ }^{2}$, N. Kaya ${ }^{3}$ \\ ${ }^{1}$ Communications Research Laboratory, 4-2-1 Koganei, Tokyo 184-8795, Japan \\ E-mail: miyake@crl.go.jp \\ 2 The Institute of Space and Astronautical Science, Yoshinodai, Sagamihara 229-8510, Japan \\ ${ }^{3}$ Kobe University, 1-1, Rokkodai, Nada, Kobe 657-8501, Japan
}

Received: 8 March 1999 / Accepted: 22 July 1999

\begin{abstract}
The occurrence frequencies of dayside ion conics with various conic angles are obtained as a function of altitude from Exos-D (Akebono) observations. We made a model calculation of ion conic evolution to match the observation results. The observed occurrence frequencies of ion conics with $80^{\circ}$ to $90^{\circ}$ conic angle are used as an input to the model and the occurrence frequencies of ion conics with smaller conic angles are numerically calculated at higher altitudes. The calculated occurrence frequencies are compared with the observed ones of ion conics with smaller conic angles. We take into account conic angle variation with altitude in both adiabatic and non-adiabatic cases, horizontal extension of ion conics due to $\mathbf{E} \times \mathbf{B}$ drift, and evolution to elevated conics and ion beams in the model. In the adiabatic case, the conic angle decreases with increasing altitude much faster than was observed. The occurrence frequency of small-angle conics is much larger than the observed value without $\mathbf{E} \times \mathbf{B}$ drift and evolution to the other UFIs. An agreement is obtained by assuming non-adiabatic variation of conic angles with altitude and an ion $\mathbf{E} \times \mathbf{B}$ drift to gyro velocity ratio of 0.08 to 0.6 , depending on geomagnetic activities.
\end{abstract}

Key words. Ionosphere (particle acceleration) . Magnetospheric physics (auroral phenomena; magnetopause, cusp, and boundary layers).

\section{Introduction}

Ion conics have cone-shaped intensity maxima centered on the magnetic field line direction in velocity space and are categorized into two types; standard conics and elevated conics. Standard conics have an angular distribution with flux maxima at a nearly constant pitch

Correspondence to: W. Miyake angle over an extended energy range (Sharp et al., 1977). The simple explanation for their generation is the perpendicular acceleration of ions at low altitudes and the subsequent upward motion of the accelerated ions in the Earth's mirror magnetic field under the conservation of the first adiabatic invariant. Elevated (or bimodal) conics (Klumpar et al., 1984) have flux maxima at a certain pitch angle at high-energy but are more fieldaligned at low-energy. Various wave-particle interaction processes have been proposed as possible mechanisms for perpendicular energization of ions (see, for a review, Andre and Yau, 1997).

Recent satellite observations suggested the heightintegrated perpendicular energization of ion conics from statistical analysis on the altitude variation of ion conics. Peterson et al. (1992) found that the average pitch angle of standard ion conics ('restricted' conics in their paper) shows a less significant variation than expected from the conservation of the first invariant at DE-1 altitudes (8000-20 $000 \mathrm{~km}$ ). Miyake et al. (1993) showed similar changes of conic angle as well as the increase of energy of ion conics with altitudes in Exos-D observations at $10000 \mathrm{~km}$. It was also revealed that a large fraction of elevated conics can be explained by height-integrated perpendicular energization of ion conics (Temerin, 1986; Miyake et al., 1996).

On the other hand, an explosive perpendicular energization of ions have been reported from sounding rocket and satellite observations (Yau et al., 1983; Andre et al., 1988; Whalen et al., 1991; Arnoldy et al., 1992; Knudsen et al., 1994). Andre et al. (1988) and Whalen et al. (1991) reported that the intense perpendicular energization of ions takes place at the equatorward edge of the cusp/cleft region. Knudsen et al. (1994) showed that the observed spatial variation of ion conics are well explained by a localized explosive energization and successive adiabatic variation with the $\mathbf{E} \times \mathbf{B}$ convection in the cusp/cleft region.

The observation of the Low Energy Particle (LEP) instrument (Mukai et al., 1990) on board Exos-D (Akebono) satellite has provided us with a large data 
set of ions and electrons over the polar region since its launch in 1989. From this data set, we can obtain the occurrence frequencies of ion conics as a function of altitude and conic angle. The purpose of this study is to examine whether the overall feature of observed occurrence frequencies of ion conics can be consistently explained by a model which considers the production of ion conics with a conic angle near $90^{\circ}$, successive conic angle variation from adiabatic to non-adiabatic cases as they flow upwards, the effect of $\mathbf{E} \times \mathbf{B}$ drift, and the possible evolution into elevated conics and ion beams, and is also to discuss what are suggested by the parameters which give an agreement to the observation results. The energization and successive evolution of ion conics are closely related to each other, and understanding of overall feature of occurrence frequencies through relevant physical processes is an important approach to identifying the ion energization and outflowing mechanisms.

There have been several reports on occurrence frequencies of ion conics based on satellite observations (Gorney et al., 1981; Yau et al., 1984; Kondo et al., 1990; Thelin et al., 1990; Peterson et al., 1992; Miyake et al., 1993, 1996). Most of them, however, did not examine the quantitative relationship of the observed occurrences between ion conics with different conic angles and at different altitudes. Miyake et al. (1993) assumed a constant production of $90^{\circ}$ ion conics at all altitudes and folding of ion conics conserving the first adiabatic invariant, and calculated a conic angle distribution at an altitude. The result showed a concentration of ion conics with a small conic angle at high altitude, but detailed occurrence frequencies for comparison were not available from the observation. In this study, observed occurrence frequencies of ion conics are available as a function of conic angle and altitude, and detailed quantitative comparisons between ion conics at different altitudes are possible.

\section{Observations}

Akebono (Exos-D) satellite was launched into a polar orbit with an initial apogee and perigee of $10482 \mathrm{~km}$ and $272 \mathrm{~km}$, respectively (Oya and Tsuruda, 1990), in 1989. The Low Energy Particle (LEP) instrument on board (Mukai et al., 1990) was designed to observe energypitch angle distributions of auroral electrons and ions. It consists of two sets of $\mathrm{E} / \mathrm{Q}$ analyzers separated at the symmetric position with respect to the satellite spin axis. The E/Q analyzers have ten detectors, sampling electrons and ions at different incident directions simultaneously and separately. It measures $10 \mathrm{eV}-16 \mathrm{keV}$ electrons and $13 \mathrm{eV}-20 \mathrm{keV}$ ions. Pitch angle distribution of charged particles is obtained by combining data from these ten detectors in a satellite spin $(7.5 \mathrm{rpm})$. The observation has been successfully carried out since the initial turn-on and a large data set for auroral electrons and ions has been built. In this study we use the data from April 1989 through April 1992.
Figure 1 shows an example of ion conics events observed during a poleward passage of dayside auroral region. The figure shows dynamic pitch angle variation with time resolution of $4 \mathrm{~s}$ for the four energy ranges. The red area represents the highest count rates of ions. One step of color code corresponds to half a decade. The white area indicates no available data in the bin. The magnetic local time was about 10:00 and the altitude was about $6000 \mathrm{~km}$.

The conics are nearly perpendicular to the magnetic field line near the low-latitude boundary of the conics region. They are folded to the field line direction at higher latitudes. The energy of conics is low near the low-latitude boundary where the conic angle is nearly $90^{\circ}$. The energy reaches the maximum where the conic angle is decreased to about $60^{\circ}$.

Most of the basic features of the conics region are explained by Knudsen et al. (1994) in terms of the polar cusp heating wall. The perpendicular energization

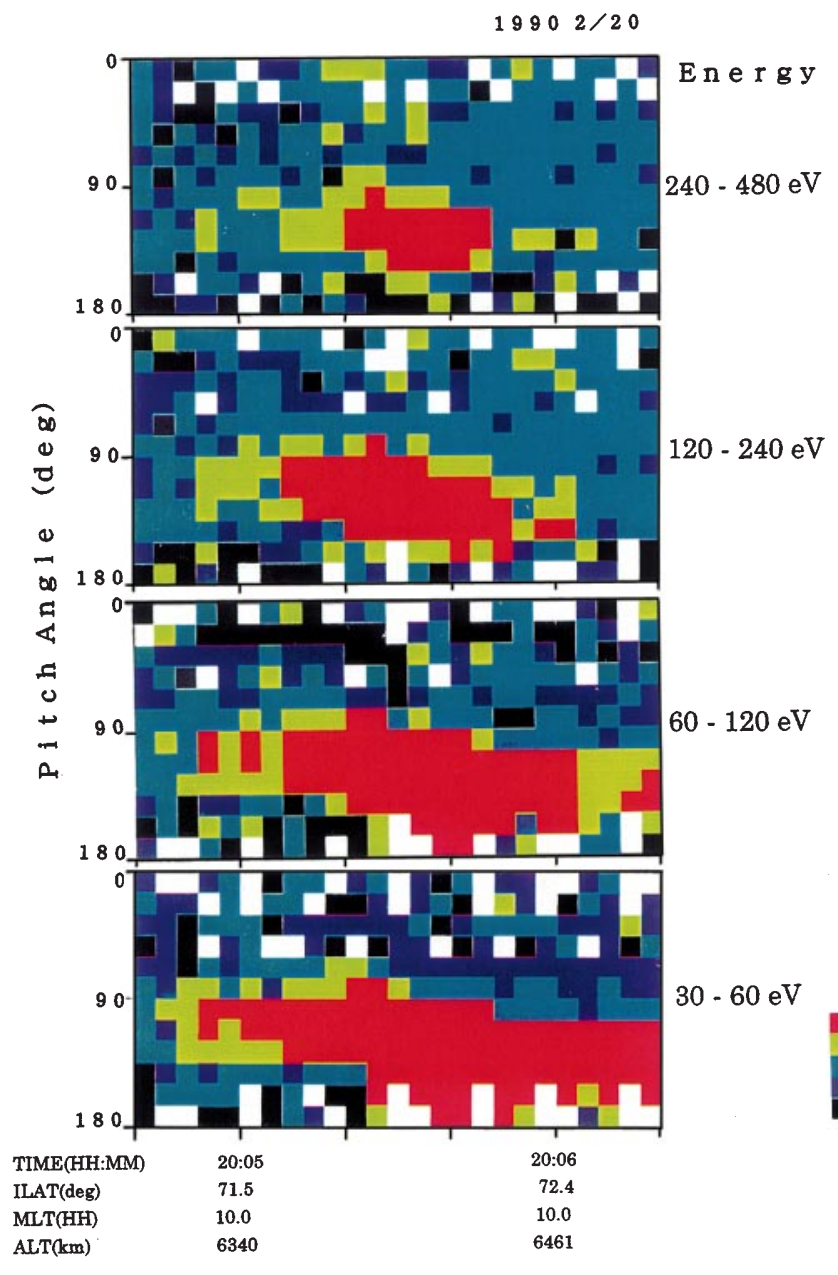

Fig. 1. An example of ion conics observed during a poleward passage of dayside auroral region. The local time was about 10:00 and the altitude was about $6000 \mathrm{~km}$. The red area represents the highest count rates. The white area shows no available data in the bin. One step of color code corresponds to half a decade. Note that the conics have a conic angle near $90^{\circ}$ and low energy near the low-latitude boundary, and a conic angle near $60^{\circ}$ and maximum energy inside the conic region 
region of ions is extended along the field line at the low latitude boundary of the cusp/cleft region. Low-energy ions are convected from low latitude to the energization region, where they are rapidly energized up to the observed energy. The ions are convected further to higher latitude as they move up to higher altitudes. At higher latitude the satellite eventually observes the ions coming from lower altitude, which results in the observed folding of ion conics with increasing latitude.

There is, however, a feature which is not explained by the simple heating wall model. The energy reaches the maximum when the conic angle is decreased to about $60^{\circ}$. Miyake et al. (1996) showed that the ion conics with conic angles near $60^{\circ}$ are statistically most energetic. This is explained by the extended energization region along the orbit of conic ions. The ion conics event in Fig. 1 represents a typical variation of ion conics in the cusp/cleft region under the influence of $\mathbf{E} \times \mathbf{B}$ drift and suggests the energization region to be extended not only along the field line but also in the latitudinal direction. The increase of energy in the lower latitude side of the conics region is attributed to the integration of perpendicular energization over the orbit of conic ions.

The decrease of energy in the higher latitude side of the conics region is basically interpreted as velocity dispersion of conic ions. All the ions move horizontally with identical $\mathbf{E} \times \mathbf{B}$ drift velocity, while the vertical velocity of ions depends on their energy and pitch angle. This leads to the observed dispersion feature of conic ions. It is well known that the same feature is also shown in precipitating ions in the cusp region (see, e.g., Mukai et al., 1991). Andre et al. (1990) undertook a twodimensional computer simulation, taking into account the $\mathbf{E} \times \mathbf{B}$ drift of ions, to model ion conics in the cusp/ cleft region and obtained a fair agreement between the observed and simulated ion distribution functions.

Figure 2 shows occurrence frequencies of standard ion conics observed by Exos-D as a function of altitude and conic angle. The occurrence frequency, $f$, is defined as $f=n / N$, where $n$ is the number of events in an AltIL-MLT (altitude - invariant latitude - magnetic local time) bin and $N$ is the number of samples in each bin. The occurrence frequencies in Fig. 2 are obtained based on observations from 0900 to 1500 MLT and within the $65^{\circ}-85^{\circ}$ invariant latitude range. The total number of events of standard conics in this MLT and IL range is 13 541. The data set and the method of ion conics identification and statistical analysis are the same as in Miyake et al. (1996). Therefore, we will not repeat them here.

The upper panel is for geomagnetically active intervals and the lower for quiet intervals, respectively. Ion conics with conic angles of $80^{\circ}$ to $90^{\circ}$ are most frequently observed near $4000-5000 \mathrm{~km}$ during active intervals and near 5000-6000 km during quiet intervals. The peak of the occurrence is shifted to smaller conic angles with increasing altitude during both active and quiet intervals. The variation with altitude is basically attributed to the folding of ion conics in the Earth's magnetic field. Another difference worth noting is that the ratio of the peak occurrence frequency of $60^{\circ}-70^{\circ}$ to
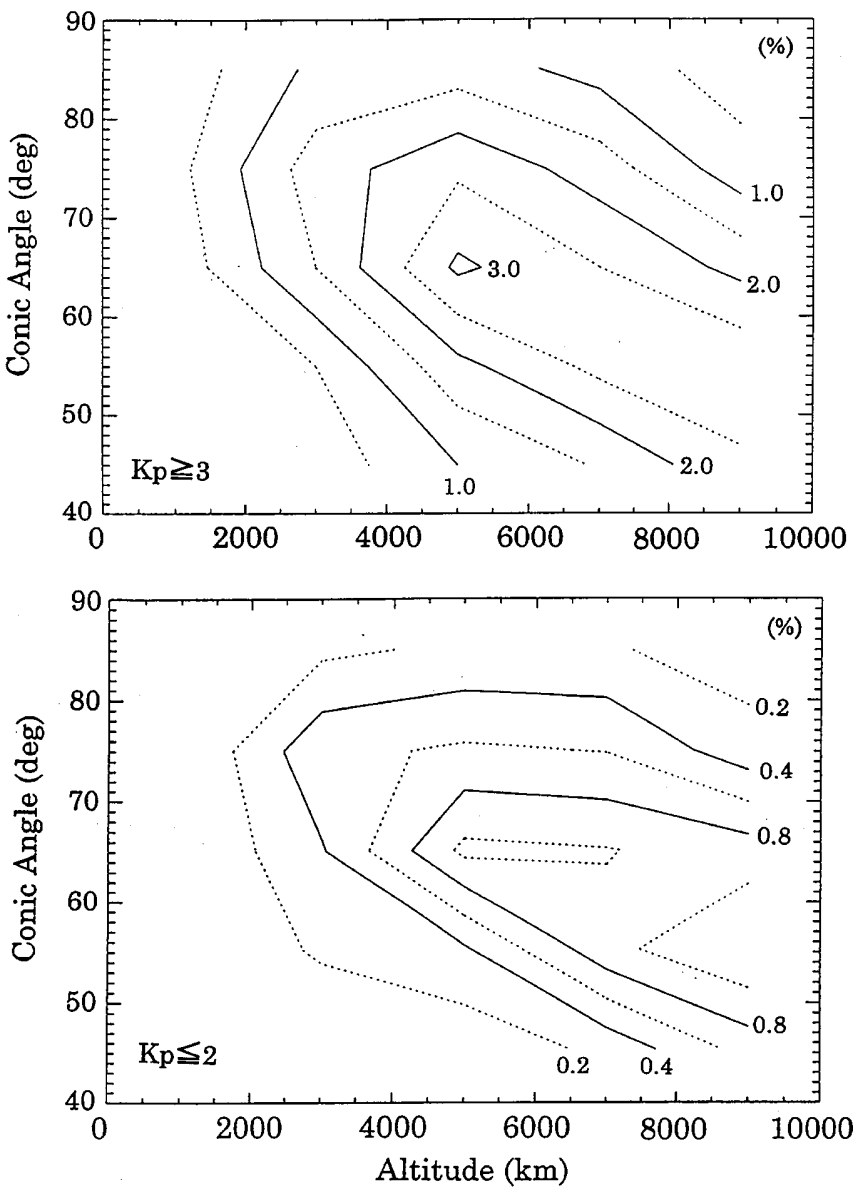

Fig. 2. The contour of the occurrence frequencies of standard ion conics as a function of altitude and conic angle observed in the cusp/ cleft region at geomagnetically active (upper panel) and quiet intervals (lower panel), respectively

that of $80^{\circ}-90^{\circ}$ is about 2.4 for the active intervals while it is about 4.4 for the quiet intervals.

\section{Method of model calculation}

As indicated from the observation, an intense perpendicular energization takes place at the equatorward edge of the cusp/cleft region and ion conics with a conic angle near $90^{\circ}$ are produced there. Our basic idea is that we may reproduce the occurrence frequencies of ion conics with smaller conic angles from a model calculation by using observed altitude distribution of the occurrence frequency of $80^{\circ}-90^{\circ}$ conics as an input of the calculation. We take a single particle approach here. We take into account the deviation of conic angle variation from the adiabatic case, the effect of ion $\mathbf{E} \times \mathbf{B}$ drift, and the evolution to the other form of UFIs in the following method.

We assume the relation of the conic angle $\alpha$ with magnetic field to be expressed as

$\sin ^{b} \alpha=\frac{B(z)}{B\left(z_{o}\right)}$

where $B(z) / B\left(z_{o}\right)$ is the ratio of the field intensity at the observation point to that at the source point (i.e., where 
$\left.\alpha=90^{\circ}\right)$. In the case of adiabatic variation, $b=2.0$. If the conics flow upward non-adiabatically in the extended region of perpendicular energization, then the folding of ion conics slows down, which can be introduced in our model by taking $b>2.0$ as a deviation from the adiabatic case.

Let us consider the case that the ions are energized in the perpendicular direction (i.e., $\alpha=90^{\circ}$ ) at point $\mathrm{A}$ in Fig. 3 . We simplify the configuration by assuming that the magnetic field line direction corresponds to the vertical direction in the figure. In the adiabatic case, the conics are folded to an angle of $80^{\circ}$ at point $\mathrm{B}, 70^{\circ}$ at $\mathrm{C}$ and $60^{\circ}$ at $\mathrm{D}$, respectively. The combination of Eq. (1) and the Earth's magnetic field model tells us that the conics are rapidly folded to the field line direction while the conic angle is large. The folding slows down when the conic angle becomes small. The ratio of the occurrence frequency of ion conics with a conic angle between $80^{\circ}$ and $90^{\circ}$ to that between $70^{\circ}$ and $80^{\circ}$ is expected to correspond to the ratio of the path length between $\mathrm{A}$ and $\mathrm{B}$ to that between $\mathrm{B}$ and $\mathrm{C}$. The ratio is smaller than unity.

When we include the effect of ion $\mathbf{E} \times \mathbf{B}$ drift in the model, the ratio above becomes larger and close to unity. The ions move from $\mathrm{A}$ to $\mathrm{D}^{\prime}$ under the drift velocity, $V_{d}$. While the conic angle is close to $90^{\circ}$ and the vertical velocity is small, ions move mostly horizontally from A to B'. When the conic angle becomes smaller and the vertical velocity becomes significant, the ions move essentially vertically from $C^{\prime}$ to $D^{\prime}$. The path length between $\mathrm{C}^{\prime}$ and $\mathrm{D}^{\prime}$ is almost equivalent to that between $\mathrm{C}$ and $\mathrm{D}$, but that between $\mathrm{A}$ and $\mathrm{B}^{\prime}$ is much larger than that between $\mathrm{A}$ and $\mathrm{B}$. Therefore, the ratio is larger than the case of no $V_{d}$, and the difference in occurrence frequencies between conics with large and small conic angles is expected to be smaller.

The effect of ion $\mathbf{E} \times \mathbf{B}$ drift is included in the model in the following way. The path length is expressed as

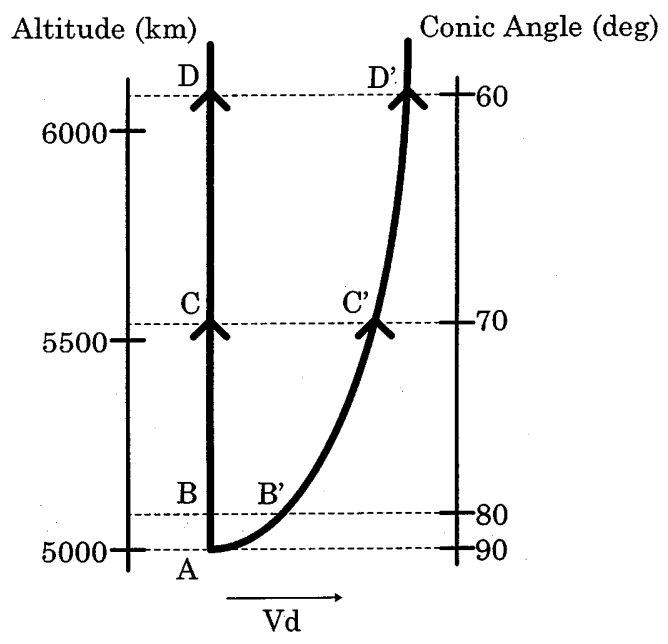

Fig. 3. A schematic drawing of the effect of ion drifts on the spatial extension of observed ion conics region. We assume that ion conics with a conic angle of $90^{\circ}$ are generated at point $A$. The ions flow up vertically due to the magnetic mirror force and move horizontally by the $\mathbf{E} \times \mathbf{B}$ drift
$L=\int \sqrt{1+\left(\frac{\mathrm{d} x}{\mathrm{~d} z}\right)^{2}} \mathrm{~d} z$

where $z$ is the altitude and $x$ is the horizontal distance. The spatial gradient of the path is given by

$\frac{\mathrm{d} x}{\mathrm{~d} z}=\frac{V_{d}}{V_{o} \cos \alpha}$

where $V_{d}$ is the horizontal drift velocity and $V_{o} \cos \alpha$ is the parallel (vertical) velocity of ions.

We also include the effect of possible evolution of standard ion conics to elevated conics and ion beams in our model. We introduce the characteristic length $L_{d}$ for evolving to the other form of UFIs. The occurrence frequency $f_{o}$ at the altitude $z$ is then reduced to

$f(z, \alpha)=\left(1.0-\frac{z-z_{o}}{L_{d}}\right) f_{o}\left(z_{o}\right)$

where $z_{o}$ is the source altitude of the conic ions (i.e., $\alpha=90^{\circ}$ ). We assume $f=0.0$ where $z-z_{o}>L_{d}$.

Let us assume a function of altitude, $f_{g}$, standing for the generation of $90^{\circ}$ conics. The occurrence frequency of ion conics with a conic angle has a single peak at a particular altitude in Fig. 2. We can introduce a simple function for $f_{g} . f_{g}$ is zero below $z_{1}$ and above $z_{4}$ and has the maximum $f_{p}$ between $z_{2}$ and $z_{3}$.

$f_{g}=f_{p} \frac{z_{o}-z_{1}}{z_{2}-z_{1}}$

between $z_{1}$ and $z_{2}$, and

$f_{g}=f_{p}-f_{p} \frac{z_{o}-z_{3}}{z_{4}-z_{3}}$

between $z_{3}$ and $z_{4}$. We choose $f_{p}, z_{1}, z_{2}, z_{3}$ and $z_{4}$ to make the calculated occurrence frequencies of ion conics with a conic angle between $80^{\circ}$ and $90^{\circ}$ as a function of altitude agree to the observation results (e.g., Fig. 2).

The occurrence frequency of ion conics as a function of altitude and conic angle is, then, given by

$F\left(z_{c}, \alpha_{c}\right)=\iint f \sqrt{1+\left(\frac{\mathrm{d} x}{\mathrm{~d} z}\right)^{2}} \mathrm{~d} z \mathrm{~d} \alpha$

where $f$ is given in Eq. (4) and $z_{c}$ and $\alpha_{c}$ are the mean of the integration interval of altitude and conic angle, respectively. The ion conics with a conic angle between $\alpha_{1}$ and $\alpha_{2}$ at the altitude of $z$ are traced back to $90^{\circ}$ conics at the source altitude between $z_{01}$ and $z_{02}$ by using Eq. (1). Therefore, we take $f_{o} \mathrm{~d} \alpha=f_{g} \mathrm{~d} z_{o}$ and use the source altitude in the actual integration.

\section{Results of model calculation}

We assume the energy of ion conics to be constant (i.e., $V_{o}=$ const.) and the drift velocity $V_{d}$ in Eq. (3) to be proportional to $r^{1.5}$ because of the Earth's dipole field where $r$ is the geocentric distance. The assumption of constant $V_{o}$ in Eq. (3) is not strictly valid when the conics flow up non-adiabatically (i.e., $b>2.0$ ). We show the calculation results of the cases in which $V_{d} / V_{o}$ is 
constant later (see Fig. 6). The later cases are equivalent to increasing the conic energy with altitude since a constant $V_{d} / V_{o}$ means that $V_{o}$ is also proportional to $r^{1.5}$. The results show that the difference between the cases of $V_{d} / V_{o} \propto r^{1.5}$ and $=$ const. is small.

Figure 4 shows the examples of calculation results in the case of adiabatic variation of conic angle $(b=2.0)$. The calculation is made for $L_{d}=\infty$ and $7000 \mathrm{~km}$, and for $V_{d}=0.0$ and $0.3 V_{o}$, respectively. The case of infinite $L_{d}$ means no evolution to the other form of UFIs. The case of zero $V_{d}$ means no horizontal drift of ions. $V_{d}$ is $0.3 V_{o}$ at $5000 \mathrm{~km}$ altitude and is proportional to $r^{1.5}$ as mentioned before.

We normalize the occurrence frequencies of conics with conic angles between $80^{\circ}$ and $90^{\circ}$ to the observation result for active intervals in the upper panel of Fig. 2, so that the occurrence has a peak around 4000 $5000 \mathrm{~km}$. The peak of occurrence is shifted gradually to higher altitude with decreasing conic angle as expected from Eq. (1). The occurrence frequency increases with decreasing conic angle and reaches more than $10 \%$ for conic angle of $40^{\circ}-50^{\circ}$ in the case of no ion drift and no evolution to the other form of UFIs (i.e., $L_{d}=\infty$ and $V_{d}=0.0$ ). This is also expected from Eq. (1) (see
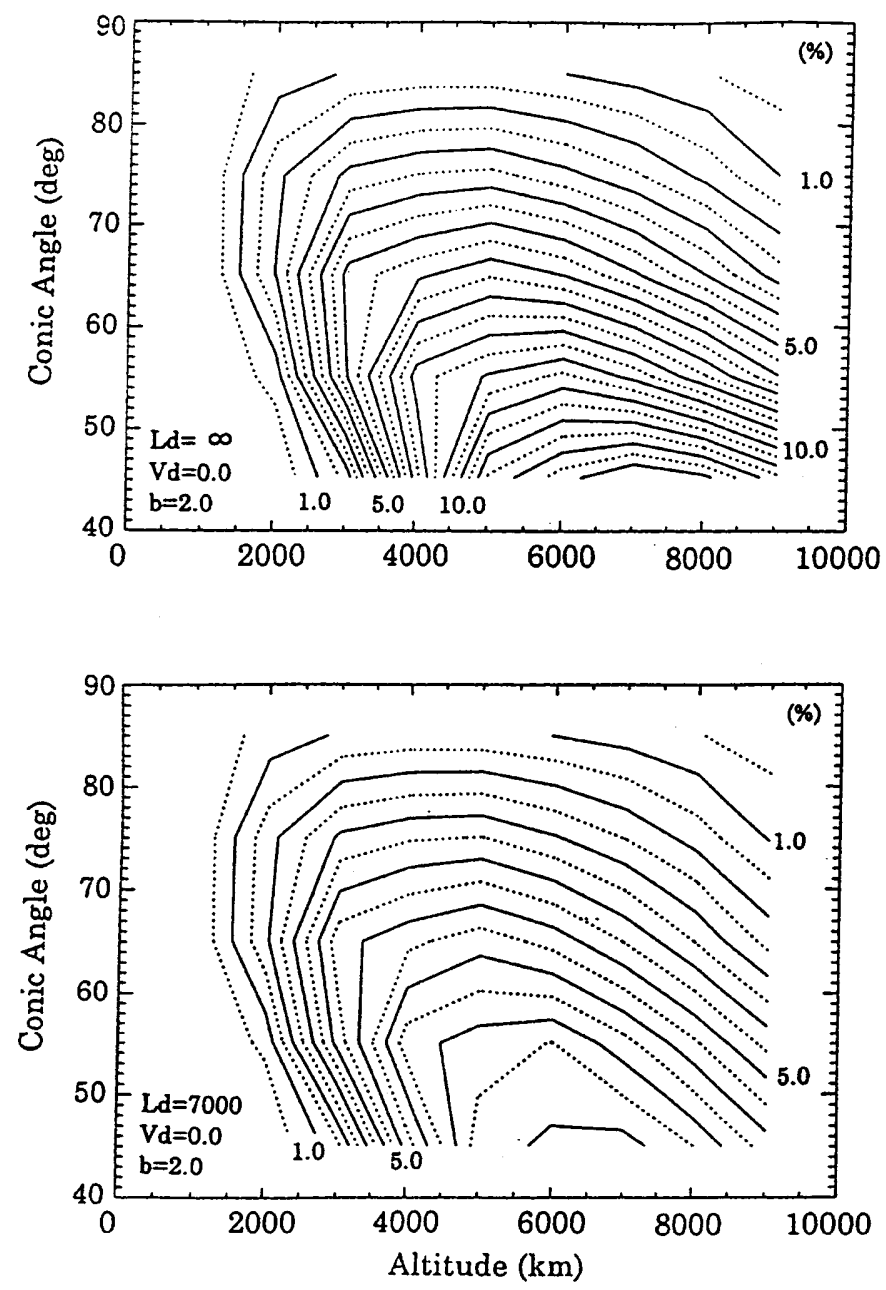

Fig. 3). The observation result, however, shows a peak of about $3 \%$ at $5000 \mathrm{~km}$ for $60^{\circ}-70^{\circ}$ conics, and no further increase of the peak value with decreasing conic angle.

It is obvious that the occurrence frequencies calculated based on simple adiabatic condition do not just fit to the observation results without the ion drift and the evolution to the other form of UFIs. We found that both the ion drift and the evolution to the other form of UFIs have the effect of reducing the occurrence frequencies at small conic angles as expected from consideration of Fig. 3. The $\mathbf{E} \times \mathbf{B}$ drift basically increases the probability of observing ion conics with a large conic angle, but it does not decrease the probability for conics with a small conic angle. Since we select $f_{p}$ in Eqs. (5) and (6) to match the occurrence frequencies of $80^{\circ}-90^{\circ}$ conics to observation, the occurrence frequency of ion conics with small conic angles is decreased with increasing $\mathbf{E} \times \mathbf{B}$ drift velocity. It is possible to reduce the occurrence frequencies at small conic angles to the observation values. The case of $L_{d}=7000 \mathrm{~km}$ and $V_{d}=0.3 V_{o}$ is an example in which the peak value of the occurrence frequencies at small conic angles are around $3-4 \%$.
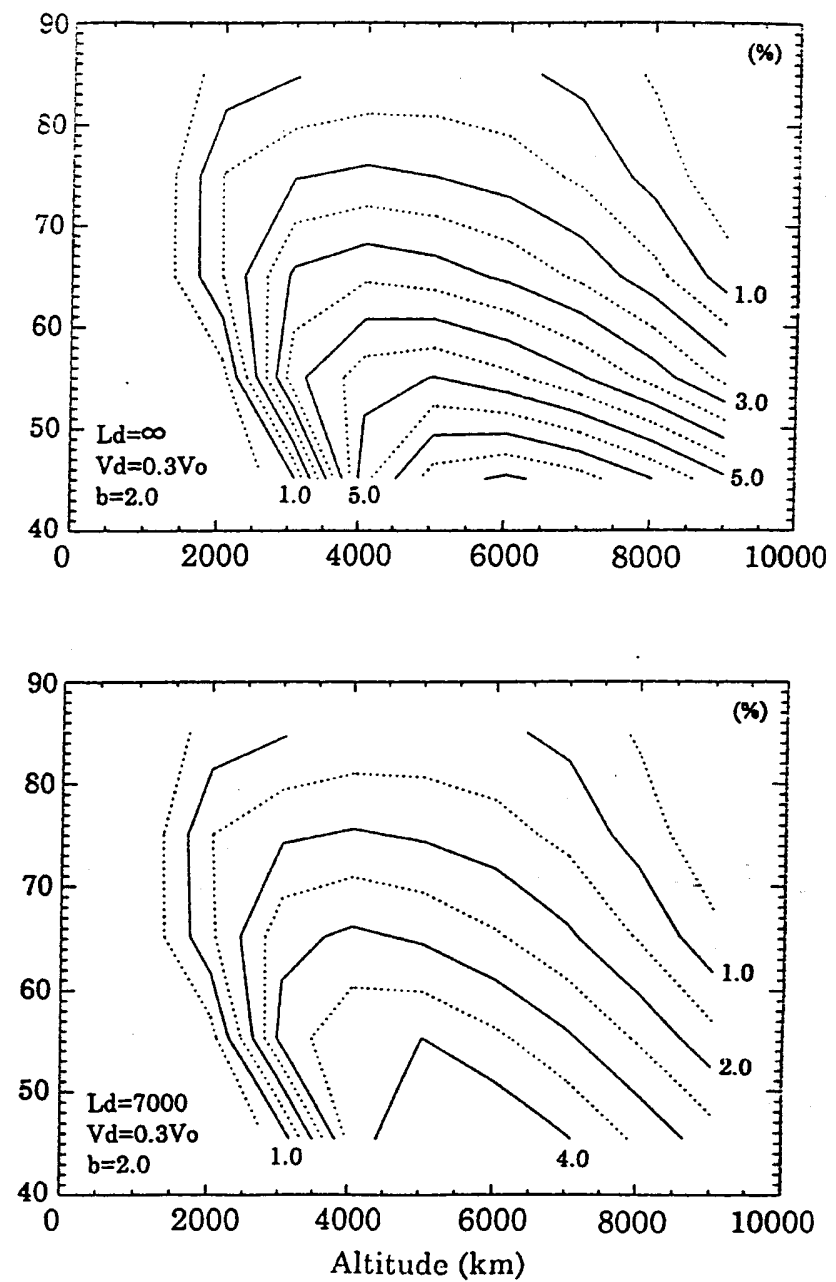

Fig. 4. Four examples of calculated occurrence frequencies as a function of altitude and conic angle under adiabatic motion $(b=2.0)$. The occurrence frequencies of conics with a conic angle of $85^{\circ}$ are adjusted to the observation results 
There remains, however, a persistent discrepancy between the observation and calculation results even when we take into account ion drift and the evolution to the other form of UFIs. The altitude of the peak occurrence frequency shows little change with decreasing conic angle in the calculation, which is due to a rapid folding of ion conics under the adiabatic assumption. The calculation results show that the peak of occurrence frequencies of $40^{\circ}-50^{\circ}$ conics is located around the altitude of $6000-7000 \mathrm{~km}$, while the observation shows that it is located above the satellite apogee (i.e., $10000 \mathrm{~km}$ ).

Figure 5 shows four examples of calculated occurrence frequencies of ion conics as a function of altitude and conic angle for the non-adiabatic case. We set $b=4.0$ in Eq. (1). The combination of $L_{d}$ and $V_{d}$ is the same as used in Fig. 4. We select here the combination of the three parameters, $L_{d}, V_{d}$, and $b$, just for the purpose of examining the basic effect of each parameter on the calculation result. We will seek an agreement to the observation result by testing various combinations of the three parameters and show the result later.

The occurrence frequencies of ion conics with small conic angles are too large without ion drift and any
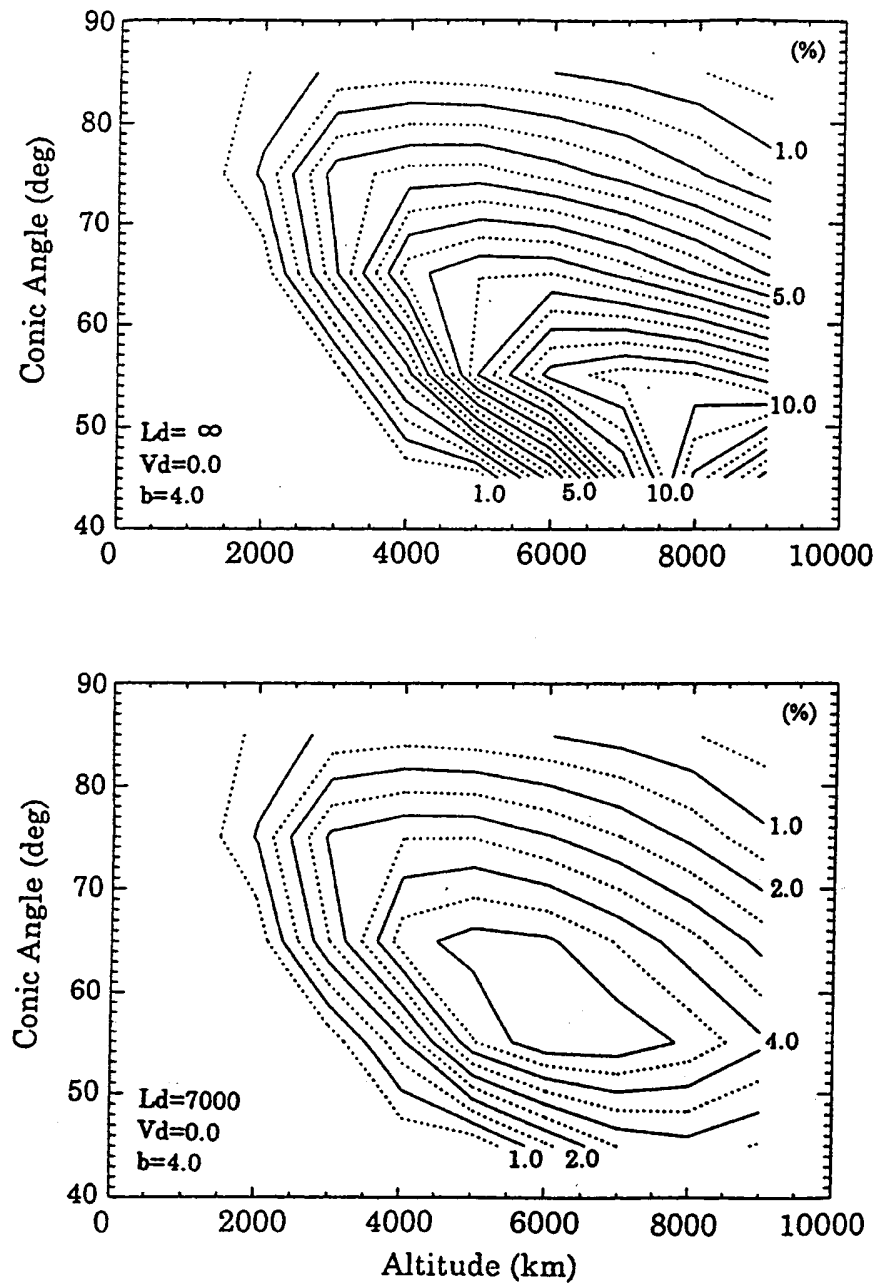

evolution to the other form of UFIs, which is basically the same as in the adiabatic cases. When we introduce a proper evolution distance and drift velocity, the peak occurrence frequency of ion conics can be around 3\% at small conic angles, almost equivalent to the observation. The clear difference from the adiabatic case is a slower change of conic angle with altitude, which causes the peak of occurrence frequency of $40^{\circ}-50^{\circ}$ conics above the satellite apogee as in observation. Conics obviously need a greater change of altitude to be folded than in the adiabatic case.

We assume that the drift velocity $V_{d}$ is dependent on the geocentric distance as $V_{d} \propto r^{1.5}$ and $V_{o}$ is constant in the calculations in Figs. 4 and 5. We take here the case of constant $V_{d} / V_{o}$ for the purpose of examining possible effect of increasing conic energy with non-adiabatic upflowing. The assumption of constant $V_{d} / V_{o}$ means $V_{o} \propto r^{1.5}$ since $V_{d}$ is independent of ion energy. It also means that ions with energy of $30 \mathrm{eV}$ at $5000 \mathrm{~km}$ are energized up to $90 \mathrm{eV}$ at $10000 \mathrm{~km}$.

The results are revealed in Fig. 6. The drift velocity is set to be $0.3 V_{o}$ at all the altitudes. The two panels in this figure are to be compared with those on the right side of Fig. 5. There is no large difference between the cases of
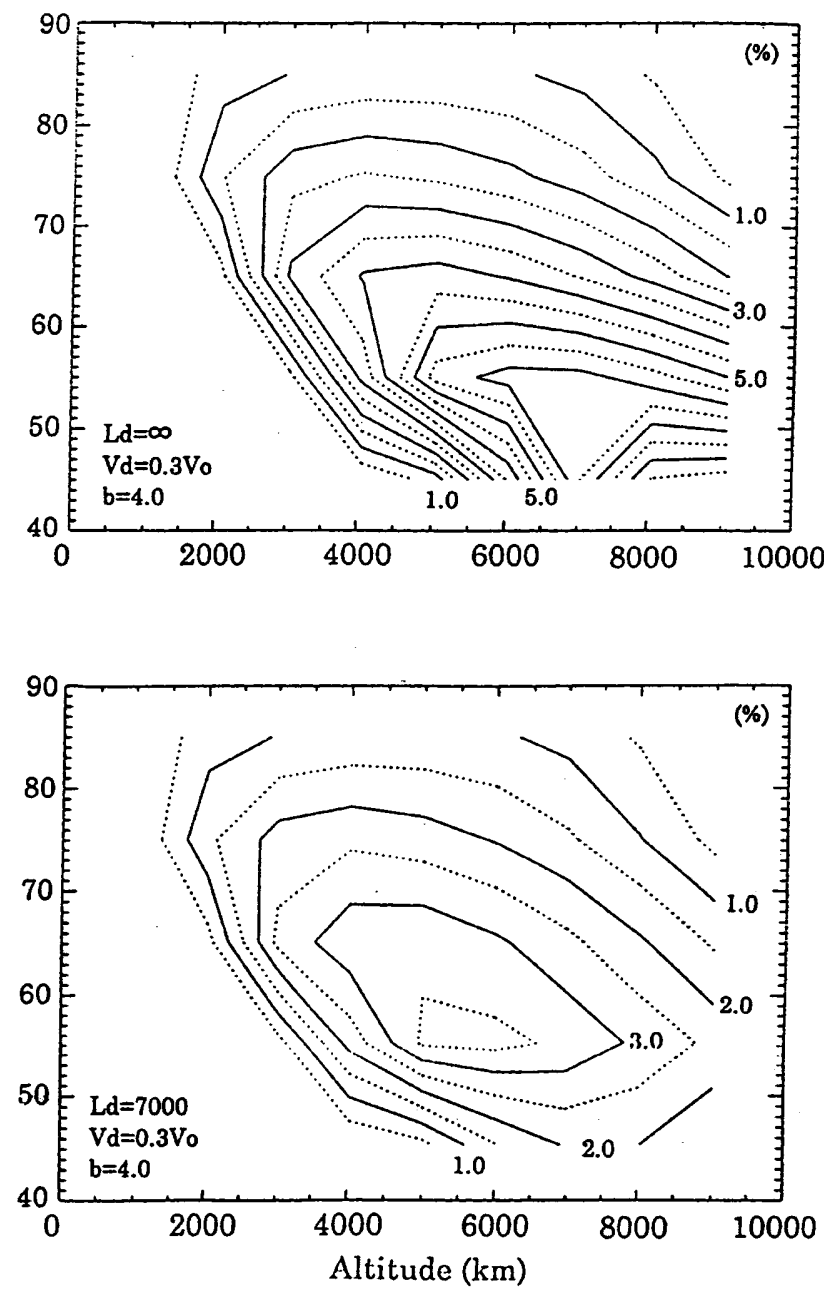

Fig. 5. Four examples of calculated occurrence frequencies as a function of altitude and conic angle under non-adiabatic motion $(b=4.0)$. The occurrence frequencies of conics with a conic angle of $85^{\circ}$ are adjusted to match the observation results 

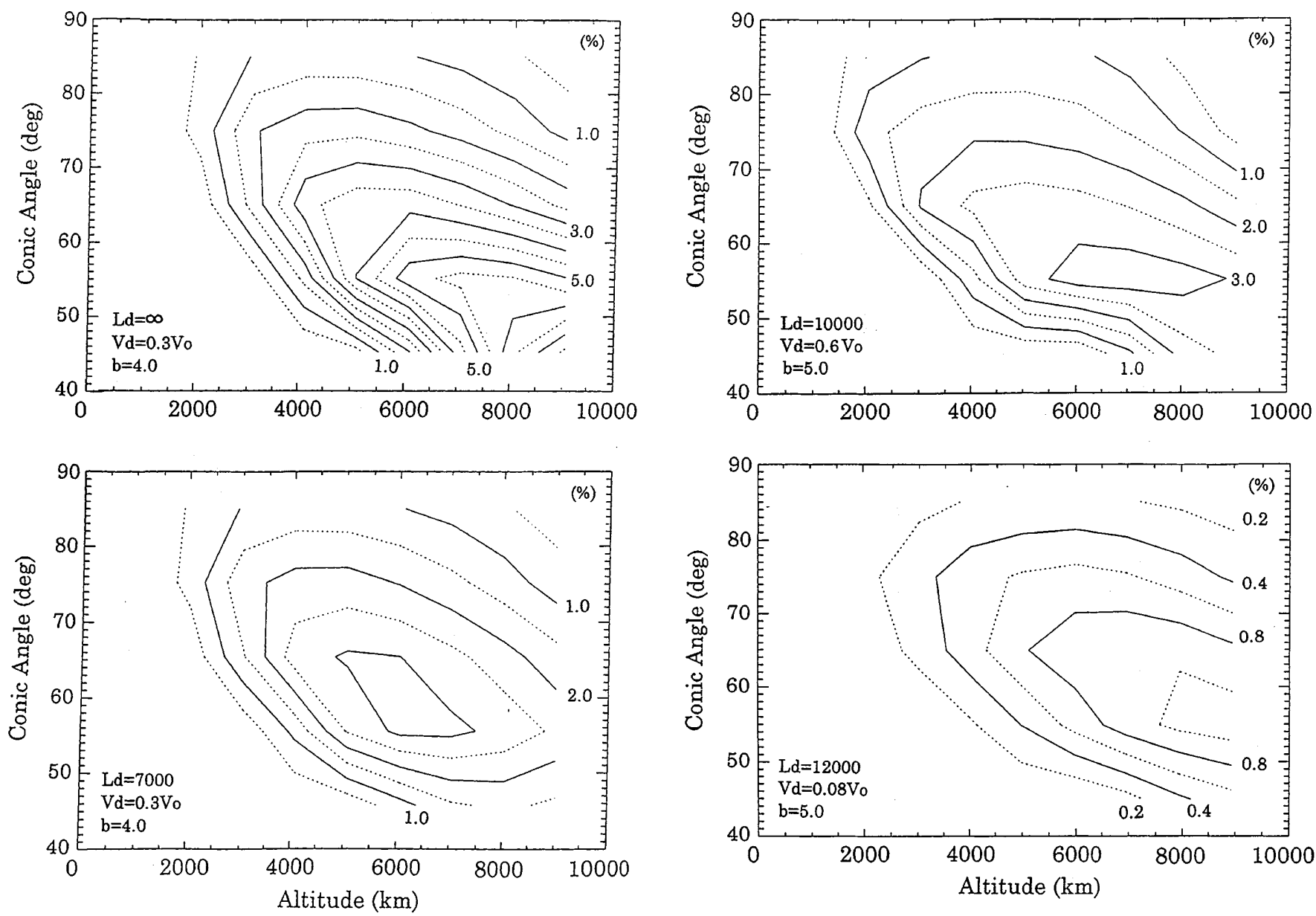

Fig. 6. Two examples of calculated occurrence frequencies as a function of altitude and conic angle. The drift velocity of ions $V_{d}$ is set to be $0.3 V_{o}$ at all the altitudes. Note that the difference from the case of the drift velocity proportional to $r^{1.5}$ (Fig. 5) is small

$V_{d} / V_{o} \propto r^{1.5}$ and of constant $V_{d} / V_{o}$, which is due to the fact that the altitude range of the calculation is not too large and we set $V_{d}=0.3 V_{o}$ at the center of the altitude range (i.e., $5000 \mathrm{~km}$ ) in both the cases.

The calculation results which are fit to the observation results in Fig. 2 well are shown in Fig. 7. The upper and lower panels are for geomagnetically active and quiet intervals, respectively. We assume here that $V_{d} / V_{o} \propto r^{1.5}$ and the values at $5000 \mathrm{~km}$ are given in the figure, again. The assumption of $V_{d} / V_{o} \propto r^{1.5}$ is not valid in the case of $b>2.0$. The difference from the case of increasing $V_{o}$ with altitude, however, is small as shown in Fig. 6 and we do not know the rate of increase of $V_{o}$ with altitude, so that we use the assumption of $V_{d} / V_{o} \propto r^{1.5}$, again.

As mentioned earlier, the increase of the peak occurrence frequency with decreasing conic angle from $80^{\circ}-90^{\circ}$ range is larger during quiet intervals than during active intervals. It leads to a larger drift velocity in the upper panel $\left(V_{d}=0.6 V_{o}\right)$ than in the lower panel $\left(V_{d}=0.08 V_{o}\right)$. When we take $\mathrm{O}^{+}$with a energy of $100 \mathrm{eV}$, then $V_{o}$ is about $25 \mathrm{~km} / \mathrm{s}$. Hence, $V_{d}$ is about $15 \mathrm{~km} / \mathrm{s}$ when $V_{d}=0.6 V_{o}$ and about $2 \mathrm{~km} / \mathrm{s}$ when $V_{d}=0.08 V_{o}$ at the altitude of $5000 \mathrm{~km}$. The $\mathbf{E} \times \mathbf{B}$ drift

Fig. 7. A set of the calculated occurrence frequencies of ion conics which fit the observation results in Fig. 2 well. The upper panel is for geomagnetically active intervals and the lower for quiet intervals, respectively

velocity of ions of these values is often observed in the cusp/cleft region (Burch et al., 1982; Mukai et al., 1991).

\section{Discussion}

Occurrence frequencies of ion conics as a function of altitude and conic angle are obtained from Exos-D observations and are compared with a model of occurrences by taking into account the effect of $\mathbf{E} \times \mathbf{B}$ drift and the evolution to the other form of UFIs. It is clear that the observation result shows slower change of conic angle with altitude than expected from the conservation of the first adiabatic invariant, as pointed out previously by Peterson et al. (1992) and Miyake et al. (1993). Neither of these authors, however, performed the quantitative comparison of occurrence frequencies with the observation.

We assumed the conic angle variation of Eq. (1), which is not necessarily valid for non-adiabatic cases. The deviation from the adiabatic variation of conic angle is expected to be large while the conic angle is large since a large conic angle generally means that the ions are still located close to the source region where 
ions are energized up to the energy range of the observation. Miyake et al. (1996) showed statistically an increase of ion energy while the conic angle is decreased from $90^{\circ}$ to $60^{\circ}-70^{\circ}$, which suggests that the ions with a large conic angle are still in the perpendicular energization region.

On the other hand, a small number of ion conics with small conic angles are found at high altitude, which are possible only when ion are energized at a very low altitude and flow up adiabatically to high altitude (Miyake et al., 1996). The deviation from the adiabatic variation of ion conics is expected to be generally small when the conic angle becomes smaller. We rather focus on the shift of the peak of occurrence frequencies with altitude in our model calculation. The shift of conic angle ranges from $80^{\circ}$ to $50^{\circ}$. Our result suggests that the variation of conic angle in Eq. (1) for the case of $b=5.0$ is a good approximation for ion conics with conic angles ranging from $80^{\circ}$ to $50^{\circ}$.

We also assumed that all the ion conics are generated at a conic angle of $80^{\circ}-90^{\circ}$. The extended energization of ions, however, suggests that a fraction of ion conics are energized up to the energy range of the observation at a conic angle of $70^{\circ}-80^{\circ}$ or even $60^{\circ}-70^{\circ}$. The simulation study of Crew et al. (1990) showed that ion conics increase their energy while the conic angle stays almost constant around $60^{\circ}$ in an extended energization region. The production of ion conics with a conic angle of $60^{\circ}$ to $80^{\circ}$ increases the occurrence frequencies of ion conics with smaller conic angles. We need a larger ratio of $\mathbf{E} \times \mathbf{B}$ drift to ion gyro velocities (i.e., $\left.V_{d} / V_{o}\right)$ to make model calculation agree with observation even if we also take into account the possible production of nonperpendicular ion conics. This may be probable since the energy of conic ions is low while the conic angle is near $90^{\circ}$. We can expect a larger $V_{d} / V_{o}$ for conic ions with a large conic angle when we take into account possible increase of ion energy.

The model calculation suggests that the $\mathbf{E} \times \mathbf{B}$ drift velocity varies with geomagnetic activity, on the basis of the observed ratio of occurrence frequencies of $80^{\circ}-90^{\circ}$ conics to those with smaller conic angles. This ratio is larger during geomagnetically quiet intervals. The actual difference of the drift velocity might be smaller since the perpendicular energization may be weaker and the production of non-perpendicular conics may be more frequent during geomagnetically quiet intervals. A further study including possible increase of ion energy and production of non-perpendicular conics is necessary for detailed comparison of the model with the observation in future.

We took a single particle analysis here and assumed that the ion with a velocity $V_{o}$ in Eq. (3) represents the entire population of ion conics. Since the velocity dispersion of conic ions due to $\mathbf{E} \times \mathbf{B}$ drift is observed (Knudsen et al., 1994 or see Fig. 1) in the cusp/cleft region, an actual ion conic is supposed to consist of ions with different sources and history. $V_{o}$ in our model should be interpreted as the typical or thermal velocity of conic ions and our result gives a good approximation when we take ions with a energy of several tens of $\mathrm{eV}$ to a few hundreds of $\mathrm{eV}$ as a typical one.

The evolution distance $L_{d}$ of $10000 \mathrm{~km}$ to $12000 \mathrm{~km}$ means that $30 \%$ to $40 \%$ of ion conics evolve to the other form of UFIs at the altitude of $9000 \mathrm{~km}$. Elevated conics are supposed to be evolved from standard ion conics through some additional energization processes (Miyake et al., 1996), and a fraction of ion beams are also generated from low-altitude ion conics by field-aligned DC electric field. The observed occurrence frequency of standard ion conics is about $6.6 \%$ at the altitude of $9000 \mathrm{~km}$ in the cusp/cleft region, while that of elevated conics is about $2.7 \%$ and that of ion beams is about $3.8 \%$ (see Miyake et al., 1996). Therefore, the evolution distance of $10000 \mathrm{~km}$ to $12000 \mathrm{~km}$ gives occurrence frequencies that are consistent with observation.

It is possible to make the calculation result similar to the observation by selecting a combination of smaller $V_{d}$ and $L_{d}$, since increasing (decreasing) $V_{d}$ has a similar effect of increasing (decreasing) $L_{d}$ on the calculation result. The occurrence frequencies of the other form of UFIs, however, are not as large as standard ion conics on the dayside (see for example, Miyake et al., 1996). A value of $V_{d}$ smaller than 10000-12000 km gives occurrence frequencies of the other form of UFIs that are too large. Therefore, the combination of $L_{d}$ and $V_{d}$ given in Fig. 7 seems most probable.

In summary, the occurrence frequencies of ion conics are obtained as a function of altitude and conic angle from Exos-D observation. The occurrence frequencies of ion conics with conic angles of $80^{\circ}-90^{\circ}$ are used as input to a model calculation and the occurrence frequencies of ion conics with smaller conic angles at a higher altitude are numerically calculated. We take into account the deviation of conic angle variation with altitude from the adiabatic case, the effect of ion $\mathbf{E} \times \mathbf{B}$ drift, and the possible evolution to the other form of UFIs. We find that the simple adiabatic model makes the conic angle decrease with altitude much faster than is observed. It is concluded that the ions flow up nonadiabatically and decrease their conic angle more slowly in the extended perpendicular energization region. The occurrence frequencies of ion conics with small conic angles become too large unless we take $\mathbf{E} \times \mathbf{B}$ drift into account. The introduction of a reasonable drift velocity, in addition to a proper evolution to the other UFIs, can decrease the occurrence frequencies of small-angle ion conics relative to those with large conic angles. It may be noted that the drift velocity, which gives an agreement to the observation results, is larger for geomagnetically active intervals and smaller for quiet intervals. The estimated drift velocities, a few to ten $\mathrm{km} / \mathrm{s}$ at $5000 \mathrm{~km}$ of altitude, are consistent with the observations in the cusp region.

Acknowledgements. We thank all the members of the Exos-D project team, especially K. Tsuruda and H. Oya, for their extensive support. We are grateful to A. W. Yau for valuable discussion and useful comments on this work.

The Editor-in-chief thanks J. L. Burch and I. Dandouras for their help in evaluating this paper. 


\section{References}

Andre, M., and A. Yau, Theories and observations of ion energization and outflow in the high latitude magnetosphere, Space. Sci. Rev., 80, 27, 1997.

Andre, M., H. Koskinen, L. Matson, and R. Erlandson, Local transverse ion energization in and near the polar cusp, Geophys. Res. Lett., 15, $107,1988$.

Andre, M., G. B. Crew, W. K. Peterson, A. M. Persoon, C. J. Pollock, and M. J. Engebretson, Ion heating by broadband low-frequency waves in the cusp/cleft, J. Geophys. Res., 95, 20 809, 1990.

Arnoldy, R. L., K. A. Lynch, P. M. Kintner, J. Vago, S. Chesney, T. E. Moore, and C. J. Pollock, Bursts of transverse ion acceleration at rocket altitudes, Geophys. Res. Lett., 19, 413, 1992.

Burch, J. L., P. H. Reiff, R. A. Heelis, J. D. Winningham, W. B. Hanson, C. Gurgiolo, J. D. Menietti, R. A. Hoffman, and J. N. Barfield, Plasma injection and transport in the mid-altitude polar cusp, Geophys. Res. Lett., 9, 921, 1982.

Crew, G.B., T. Chang, S. M. Retterer, W. K. Peterson, D. G. Gurnett, and R. L. Huff, Ion cyclotron resonance heated conics: theory and observations, J. Geophys. Res., 95, 3959, 1990.

Gorney, D. J., A. Clarke, D. Croley, J. Fennel, J. Luhmann, and P. Mizera, The distribution of ion beams and conics below 8000 km, J. Geophys. Res., 86, 83, 1981.

Klumpar, D. M., W. K. Peterson, and E. G. Shelley, Direct evidence for two-stage (bimodal) acceleration of ionospheric ions, J. Geophys. Res., 89, 10 779, 1984.

Knudsen, D. J., B. A. Whalen, T. Abe, and A. W. Yau, Temporal evolution and spatial dispersion of ion conics: evidence for a polar heating wall, in Solar System Plasmas in Space and Time, Geophys. Monogr. vol 84, eds. J. L. Burch and J. H. Waite, 163 169, AGU, Washington, D.C., 1994.

Kondo, T., B. A. Whalen, A. W. Yau, and W. K. Peterson, Statistical analysis of upflowing ion beams and conics distributions at DE1 altitudes, J. Geophys. Res., 95, 12 091, 1990.
Miyake, W., T. Mukai, and N. Kaya, On the evolution of ion conics along the field line from EXOS D observations, J. Geophys. Res., 98, 11 127, 1993.

Miyake, W., T. Mukai, and N. Kaya, On the origins of the upward shift of elevated (bimodal) ion conics in velocity space, J. Geophys. Res., 101, 26 961, 1996.

Mukai, T., et al., Low energy charged particle observations in the 'auroral' magnetosphere: first results from Akebono (Exos-D) satellite, J. Geomag. Geoelectr., 42, 479, 1990.

Mukai, T., A. Matsuoka, H. Hayakawa, S. Machida, K. Tsuruda, and A. Nishida, Signatures of solar wind injection and transport in the dayside cusp: Exos-D observations, Geophys. Res. Lett., 18, 333, 1991.

Oya, H., and K. Tsuruda, Introduction to the Akebono (Exos-D) satellite observations, J. Geomag. Geoelectr., 42, 367, 1990.

Peterson, W. K., H. L. Collin, M. F. Doherty, and C. M. Bjorklumd, $\mathrm{O}^{+}$and $\mathrm{He}^{+}$restricted and extended (bi-modal) ion conic distributions, Geophys. Res. Lett., 19, 1439, 1992.

Sharp, R. D., R. G. Johnson, and E. G. Shelley, Observations of an ionospheric acceleration mechanism producing energetic $(\mathrm{keV})$ ions primarily normal to the geomagnetic field direction, J. Geophy. Res., 82, 3324, 1977.

Temerin, M., Evidence for a large bulk ion conic heating region, Geophys. Res. Lett., 13, 1059, 1986.

Thelin, B., B. Aparicio, and R. Lundin, Observations of upflowing ionospheric ions in the mid-altitude cusp-cleft region with the VIking satellite, J. Geophys. Res., 95, 5931, 1990.

Whalen, B. A., S. Watanabe, and A. W. Yau, Observations in the transverse ion energization region, Geophys. Res. Lett., 18, 725, 1991.

Yau., A. W., B. A. Whalen, A. G. Mcnamara, P. J. Kellog, and W. Bernstein, Particle and wave observations of low-altitude ionospheric ion acceleration events, J. Geophys. Res., 88, 341, 1983.

Yau, A. W., B. A. Whalen, W. K. Peterson, and E. G. Shelley, Distribution of upflowing ionospheric ions in the high-altitude polar cap and auroral ionosphere, J. Geophys. Res., 89, 5507, 1984. 